\title{
Deep Generative Model with Supervised Latent Space for Text Classification
}

\author{
Maciej Jankowski ${ }^{1, *}$ \\ ${ }^{1}$ Faculty of Cybernetics, Military University of Technology in Warsaw
}

\begin{abstract}
Recent advances in applying deep neural networks to Bayesian Modelling, sparked resurgence of interest in Variational Methods. Notably, the main contribution in this area is Reparametrization Trick introduced in [1] and [2]. VAE model [1], is unsupervised and therefore its application to classification is not optimal. In this work, we research the possibility to extend the model to supervised case. We first start with the model known as Supervised Variational Autoencoder that is researched in the literature in various forms [3] and [4]. We then modify objective function in such a way, that latent space can be better fitted to multiclass problem. Finally, we introduce a new method that uses information about classes to modify latent space, so it even better reflects differences between classes. All of this, will use only two dimensions. We will show, that mainstream classifiers applied to such a space, achieve significantly better performance than if applied to original datasets and VAE generated data. We also show, how our novel approach can be used to calculate better classification score, and how it can be used to generate data for a given class.
\end{abstract}

\section{Introduction}

In this paper, we propose and analyze two new models that are based on previous results in Bayesian Neural Networks modeling. Both models extend standard VAE by adding response variable, that is used for class label prediction. Instead of using Kullback-Leibler divergance to regularize the model, we will introduce a cost function, that forces latent space to behave as if it came from mixture of Gaussian distributions. The difference between those two models is, that in the first case, we do not supervise latent space, while in the second case, we do. The supervision in the second case means, that for each data point in latent space, we are explicitly providing information, from which mixture component this point is expected to have been generated. The rest of the paper is structured as follows: in the second section we introduce the concept of Supervised Variational Autoencoder (VAE), the third section modifies this model, by replacing KL term with cost that forces points in latent space to be clustered according to some specially chosen Gaussian distributions. The fourth section extends this approach, by explicitly assigning points from single class to a single Gaussian. The fifth section focuses on applications of the model and the last sixth section contains results of experiments. In section 7 , we summarize the results.

\subsection{Notation}

Let $C$ be the number of classes, $P$ and $J$ be the dimensionality of input documents and hidden variables respectively. Let $M$ be the number of samples (documents). Random variables $x \in \mathcal{R}^{P}, y \in \mathcal{R}$ and $z \in R^{J}$ denote document, class

*e-mail: maciej.jankowski@wat.edu.pl and hidden variable. When we add a superscript, e.g. $x^{(d)}$, $y^{(d)}$ and $z^{(d)}$, we mean a concrete realization of random variables.

\section{Supervised Variational Autoencoder}

Our goal is to reduce dimensionality of texts in such a way, that we can efficiently classify them. We will now introduce the concept of Supervised Variational Autoencoder and show, that this approach can achieve much better performance in terms of classification accuracy than VAE model. The model is implemented as a neural network, where the left part of the network encodes parameters of posterior $q_{\phi}(z \mid x)=\mathcal{N}(\mu(\phi), \Sigma(\phi))$, where $\mu(\phi) \in \mathcal{R}^{J}$ and $\Sigma(\phi)) \in \mathcal{R}^{J \times J}$. The right part of the network encodes distribution of reconstruction $p_{\theta}(x \mid z)=\mathcal{B} \operatorname{er}(b(\theta))$ and a distribution of a class $p_{\theta}(y \mid z)=\operatorname{Cat}(\tau(\theta))$. In this settings, parameters $b(\theta)$ and $\tau(\theta)$ are calculated by neural network. The generative process of SVAE is as follows:

1. Draw $z \sim \mathcal{N}(0, I)$

2. Draw $x \mid z, \theta \sim \mathcal{B} \operatorname{er}(b(\theta))$,

3. Draw $y \mid z, \theta \sim \operatorname{Cat}(\tau(\theta))$,

where $\theta$ are model parameters, $\operatorname{Ber}(b(\theta))$ is a Bernoulli distribution, and $C \operatorname{at}(\tau(\theta))$ is a categorical distribution, that is, multinomial distribution with a single trial and probability of success of each outcome described by vector $\tau(\theta)$. The graphical representation of the model is given in the form of plate notation in figure 1. As we can see, both $x$ and $y$ depend on $z$. Variables $x, y$ and $z$, also depend on parameters $\theta$. Prior distribution of $z$ is $\mathcal{N}(0, I)$, and true posterior distribution is $p_{\theta}(z \mid x)$, therefore it also depends on $\theta$. Solid lines denote the generative model $p(z) p_{\theta}(x \mid z) p_{\theta}(y \mid z)$. 


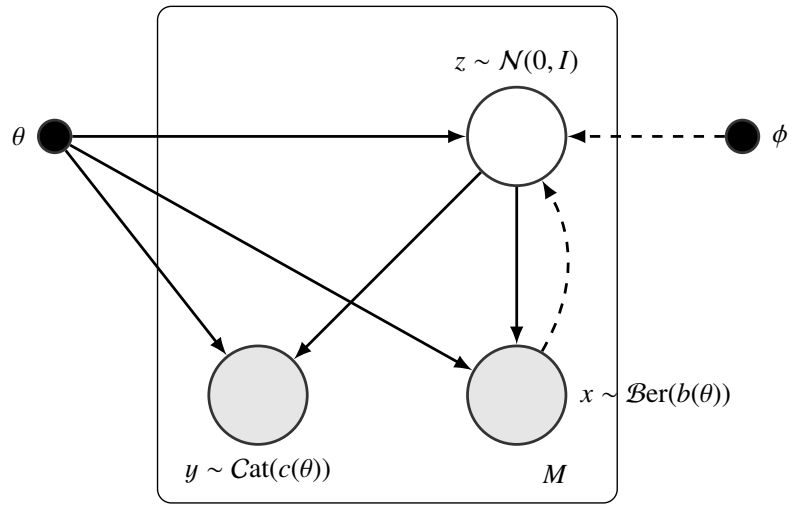

Figure 1: Generative process of Supervised Variational Autoencoder

Dashed lines denote the variational approximation $q_{\phi}(z \mid x)$ to intractable posterior $p_{\theta}(z \mid x)$. Index $M$, in the bottom right corner means, that this process is executed $M$ times, once for each document.

\subsection{Cost function}

As shown in the figure 1, we make the simplifying assumption, that $x \perp y \mid z$. We assume, that everything we know about the class can be captured by hidden variables. In other words, knowing the state of $z$, values of $x$ do not provide any additional information for predicting $y$. As usual, we start with $\operatorname{KL}\left(q_{\phi}(z \mid x) \| p_{\theta}(z \mid x, y)\right)$, to finally arrive at $E L B O$

$$
\begin{aligned}
\mathrm{KL}\left[q_{\phi}(z \mid x) \| p_{\theta}(z \mid x, y)\right] & =\mathbb{E}_{z \sim q_{\phi}}\left[\log q_{\phi}(z \mid x)\right. \\
& \left.-\log p_{\theta}(x, y \mid z)-\log p(z)\right] \\
& +\log p_{\theta}(x, y)
\end{aligned}
$$

then

$$
\begin{aligned}
\log p_{\theta}(x, y)-\mathrm{KL}\left[q_{\phi}(z \mid x) \| p_{\theta}(z \mid x, y)\right] & =\mathbb{E}_{z \sim q_{\phi}}\left[\log p_{\theta}(x, y \mid z)\right] \\
& -\operatorname{KL}\left[q_{\phi}(z \mid x) \| p(z)\right]
\end{aligned}
$$

Using $x \perp y \mid z$, we can write

$$
\begin{aligned}
\log p_{\theta}(x, y) & -\operatorname{KL}\left[q_{\phi}(z \mid x) \| p_{\theta}(z \mid x, y)\right]=\mathbb{E}_{z \sim q_{\phi}}\left[\log p_{\theta}(x \mid z)\right] \\
& +\mathbb{E}_{z \sim q_{\phi}}\left[\log p_{\theta}(y \mid z)\right]-\operatorname{KL}\left[q_{\phi}(z \mid x) \| p(z)\right]
\end{aligned}
$$

As in VAE, the last term of the RHS is easy to compute. We assume, that the posterior distribution of hidden variables $p_{\theta}(z \mid x)$ is normally distributed. We define a family of functions $q_{\phi}(z \mid x)=\mathcal{N}(\mu(\phi), \Sigma(\phi))$, that approximate $p_{\theta}(z \mid x)$. The family is parameterized by variational parameters $\phi$. The choice of values for $\phi$ determine the quality of approximation. Following [1], we use a deep neural network for encoder $q_{\phi}(z \mid x)$.

In practice, we would like to control how much weight do we put into classification $\log p_{\theta}(y \mid z)$, as opposed to reconstruction $\log p_{\theta}(x \mid z)$ and regularization $\mathrm{KL}\left[q_{\phi}(z \mid x) \| p(z)\right]$. We introduce parameters $\lambda, \kappa$ and $\rho$, that can modify behavior of the algorithm. The final optimization problem is

$$
\begin{aligned}
\left(\theta^{*}, \phi^{*}\right) & =\underset{\theta, \phi}{\operatorname{argmax}}\left\{\lambda \mathbb{E}_{z \sim q_{\phi}}\left[\log p_{\theta}(x \mid z)\right]\right. \\
& \left.+\kappa \mathbb{E}_{z \sim q_{\phi}}\left[\log p_{\theta}(y \mid z)\right]-\rho \operatorname{KL}\left[q_{\phi}(z \mid x) \| p(z)\right]\right\}
\end{aligned}
$$

\subsection{Latent space}

In figure 4, we present visualization of latent space for SVAE model. Each point $z^{(d)}$ on the scatter plot is a sample from two dimensional $z \sim q_{\phi}(z \mid x)$. Parameters in equation 1 were set to $\lambda=0, \kappa=1$ and $\rho=0.5$. When $\lambda=0$, separation between classes is the most visible. Depending on the application of the model, we should choose some intermediate value between 0 and 1 . For example, for training purposes, it is better to allow for higher variation between examples to increase generalization. Another parameter $\rho$, controls how much do we regularize the model with $K L$ term. If the model is not regularized, then weights (and as a results vales of $z$ ), become large (see figure 5 (Right)). If, on the other hand, $\rho$ is to large, then separation of classes in hidden layer disappears, and all values of $z$, look as if they came from $\mathcal{N}(0, I)$.

\section{Deep generative model with GMM latent space}

Supervised Variational Autoencoder use $p(z)=\mathcal{N}(0, I)$ as a prior for latent space. Therefore, $\operatorname{KL}\left[q_{\phi}(z \mid x) \| p(z)\right]$ acts as a regularization component that pushes values of $z$ towards the center of the coordinate system. If we remove the $K L$ component, the latent space looks differently. Both latent spaces are visualized in figure 5. Additionally, the classifier with unconstrained latent space achieves better results. This suggests, that forcing latent space to behave as if it came from $\mathcal{N}(0, I)$ may not be the best choice. The natural candidate is a mixture of distributions, with the number of components equal to the number of classes. In this chapter, we focus our attention on a mixture of Gaussians (GMM) as a latent space. We will call this model Deep Latent GMM model (DLGMM).

Gaussian Mixture Model is a superposition of $K$ Gaussian densities of the form

$$
g(z)=\sum_{k=1}^{K} \pi_{k} \mathcal{N}\left(z \mid \mu_{k}, \Sigma_{k}\right), \quad \sum_{k=1}^{K} \pi_{k}=1, \quad 0 \leq \pi_{k} \leq 1,
$$

where $\pi \in \mathcal{R}^{K}$, are mixing coefficients and $\mu_{k} \in \mathcal{R}^{J}, \Sigma_{k} \in$ $\mathcal{R}^{J \times J}, k=1, \ldots, K$, are means and covariances of mixture components. We will describe the exact derivation of those parameters in section 3.1. For GMM model, we define $K$ dimensional binary random variable $s$. This variable, has 1 of $K$ representation (one-hot). Only one element of $s_{k}$ is equal to 1 and all other elements are equal to 0 . We can write $s_{k} \in\{0,1\}$ and $\sum_{k=1}^{K} s_{k}=1$. The marginal distribution of $s$ is expressed in terms of mixing coefficients $\pi_{k}$

$$
p\left(s_{k}=1\right)=\pi_{k}
$$


For observed dataset of $M$ datapoints, realization of $s$ is a $M \times K$ matrix. Therefore, each element will be indexed with two indices $s_{k}^{(d)}, d=1, \ldots, M, k=1, \ldots, K$. Mixture components will be evenly spaced on a circle, as in figure 6. The exact derivation of the parameters of those Gaussians is summarized in the next section.

\subsection{Generate 2D Gaussians on a circle}

Our goal is to automatically generate Gaussian distributions in $2 D$, that are spread evenly on a circle. We start with some initial distribution and successively progress along the line of a circle and rotate "ellipse" of the next Gaussian distribution. In our experiments, the starting distribution is

$$
\mathcal{N}\left(\mu=[1,0], \Sigma=\left[\begin{array}{cc}
0.07 & 0 \\
0 & 0.007
\end{array}\right]\right)
$$

To generate $K$ distributions around the circle, we follow the procedure in algorithm 1. The figure 6 shows, how samples from those distributions look like.

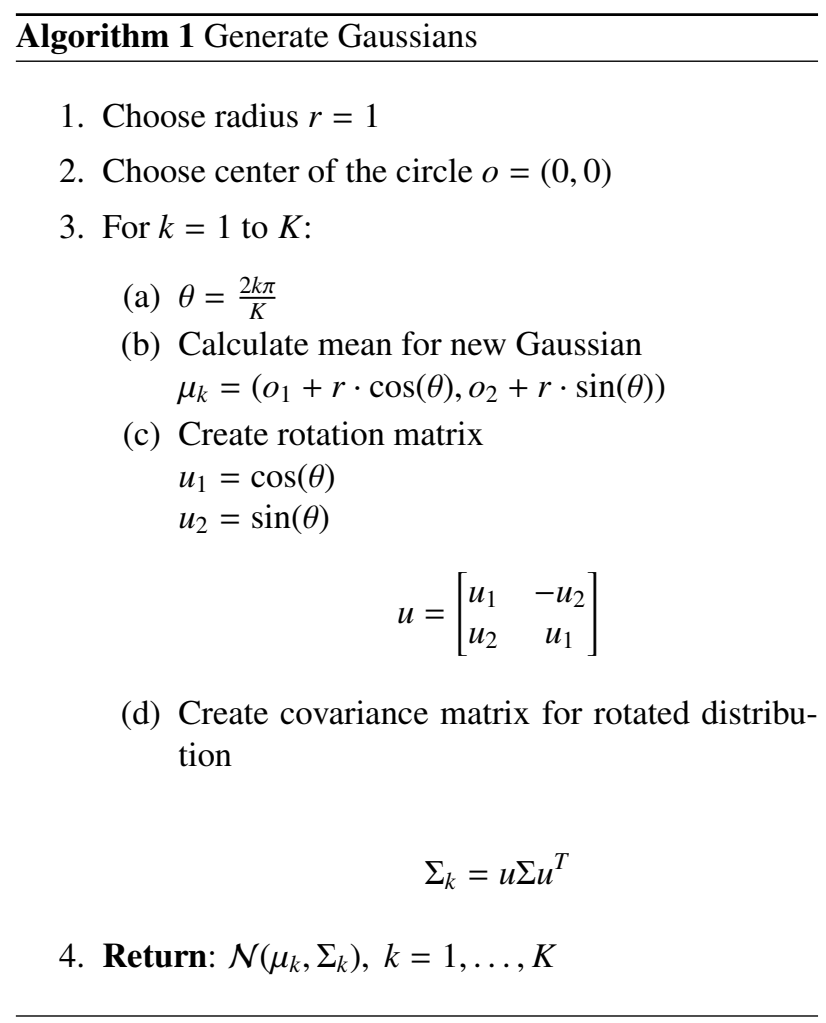

\subsection{Generative process}

In this section, we present the generative process of DLGMM. Let $K$ be the number of distributions in mixture. Assuming, that parameters $\pi, \mu$ and $\Sigma$ are given, each document and its label is generated according to the following process

1. Draw $s \sim \operatorname{Cat}(\pi)$

2. Draw $z \sim \mathcal{N}\left(\mu_{s}, \Sigma_{s}\right)$

3. Draw $x \mid z, \theta \sim \mathcal{B} \operatorname{er}(b(\theta))$,

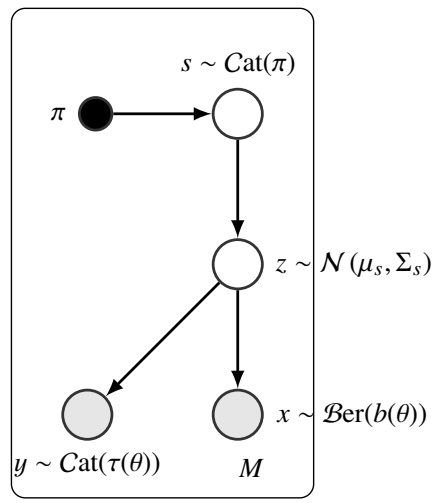

Figure 2: Generative process of Deep generative model with GMM latent space

\section{Draw $y \mid z, \theta \sim \operatorname{Cat}(\tau(\theta))$,}

where $\operatorname{Ber}(b(\theta)))$ is a Bernoulli distribution, $\operatorname{Cat}(\tau(\theta))$ is a categorical distribution and $\pi$ are mixture coefficients that constitute a prior distribution of mixture components. Generative process is presented in the form of a plate diagram in the figure 2 .

\subsection{Cost function}

For our purposes, we will often assume, that $K=C$. Our goal is to modify the cost function in such a way, that the distribution of points in latent space follow mixture of multivariate Gaussian distributions. The density function of $z$ is given by 2 The log likelihood function that we want to maximize is just a logarithm of product of those functions (we assume that samples $z^{(1)}, \ldots, z^{(M)}$ are taken independently from the same distribution). Since we want to use this function as a penalty for not following mixture of Gaussians, we are taking negative value of the loglikelihood

$$
\Omega(z)=-\sum_{d=1}^{M} \ln \left[\sum_{k=1}^{K} \pi_{k} \mathcal{N}\left(z^{(d)} \mid \mu_{k}, \Sigma_{k}\right)\right] .
$$

Gradient of 3 is

$$
\frac{\partial \Omega}{\partial z^{(d)}}=\sum_{k=1}^{K} \gamma_{k}\left(z^{(d)}\right) \Sigma_{k}^{-1}\left(z^{(d)}-\mu_{k}\right),
$$

where

$$
\gamma_{k}\left(z^{(d)}\right)=\frac{\pi_{k} \mathcal{N}\left(z^{(d)} \mid \mu_{k}, \Sigma_{k}\right)}{\sum_{k=1}^{K} \pi_{k} \mathcal{N}\left(z^{(d)} \mid \mu_{k}, \Sigma_{k}\right)} .
$$

Having defined the penalty term for latent space, we can now formulate our objective function

$$
\begin{aligned}
\mathcal{L}_{\phi, \theta}(x, y)=\lambda \mathbb{E}_{z \sim q_{\phi}}\left[\log p_{\theta}(x \mid z)\right] & +\kappa \mathbb{E}_{z \sim q_{\phi}}\left[\log p_{\theta}(y \mid z)\right] \\
& -\rho \Omega(z) .
\end{aligned}
$$

Optimization problem is

$$
\left(\theta^{*}, \phi^{*}\right)=\underset{\theta, \phi}{\operatorname{argmax}} \mathcal{L}_{\phi, \theta}(x, y) .
$$


It is important to note, that our model no longer follows Variational Inference principle. Additionally, we do not show, that it is a lower bound of likelihood function $p_{\theta}(x)$. We will show however, that it has some usefull characteristics compared to VAE model.

\section{Deep Generative Models with Supervised GMM Latent Space}

Although shaping latent space as a mixture of Gaussians seems to be a better choice than $\mathcal{N}(0, I)$, we can identify at least two inconveniences with this approach.

Firstly, in some cases, classes may not be assigned to separate Gaussians. It may happen, that points for two different classes end up being distributed according to a single mixture component. It may also happen, that one of the classes is so similar to more than one other class, that its representation in the latent space is located in the center of coordinate system. This way, it is close to all other points.

Secondly, our data set may be unbalanced. It would have been very convenient, if we could use a prior knowledge about classes distribution to provide proper weights for mixture components in the form of mixing coefficients $\pi_{1}, \ldots, \pi_{K}$. However, latent space is found in completely unsupervised fashion. We do not provide any information which Gaussian should represent which class. The only thing that we require is, that latent space is distributed according to mixture of Gaussians. In this section, we research the idea of supervising the training process of the latent space. We will call our new model Deep Supervised Latent GMM model (DSLGMM).

\subsection{Generative process}

The difference between DLGMM and DSLGMM is, that $s$ is no longer a hidden variable, but its value is observed. Usually $s=\sigma(y)$, where $\sigma$ is a permutation of labels. Let $\theta$ be the model parameters. For given values $s, \mu$ and $\Sigma$, document $x$ and its label $y$ are created in the following way

1. Draw $z \mid s \sim \mathcal{N}\left(\mu_{s}, \Sigma_{s}\right)$

2. Draw $x \mid z, \theta \sim \mathcal{B} \operatorname{er}(b(\theta))$,

3. Draw $y \mid z, \theta \sim \operatorname{Cat}(\tau(\theta))$,

Graphical representation of this process is presented in the figure 3.

\subsection{Cost function}

We set a number of mixing components to the number of classes $C=K$ and we will use one-hot-encoded label index as the value for $s$. Therefore, we can assume, that values of $s$ are observed. Now, since index of cluster $s$ is observed, we can work with joint probability $p(z, s)$. For $M$ observed data-points and its labels, likelihood is given by

$$
p(z, s)=\prod_{k=1}^{K} \pi_{k}^{s_{k}} \mathcal{N}\left(z \mid \mu_{k}, \Sigma_{k}\right)
$$

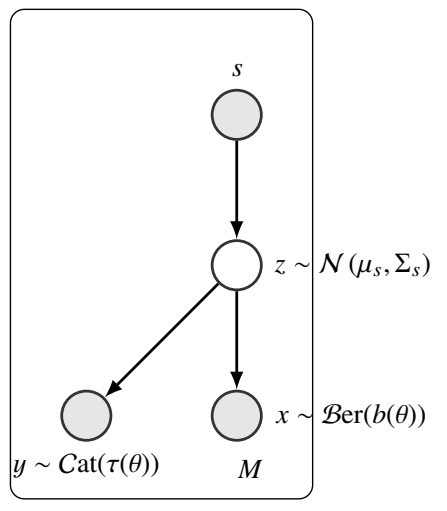

Figure 3: Generative process of DSLGMM

Log likelihood is

$$
\ln p(z, s)=\sum_{k=1}^{K} s_{k}\left[\ln \pi_{k}+\ln \mathcal{N}\left(z \mid \mu_{k}, \Sigma_{k}\right)\right]
$$

where $s$ is defined for $d$-th document and $s_{k}$ is a $k$-th value of $s$, that is 0 or 1 . Gradient of this function is

$$
\frac{\partial}{\partial z} \ln p(z, s)=\sum_{k=1}^{K} s_{k} \Sigma^{-1}(z-\mu) .
$$

The penalty function is therefore defined as $\Gamma(z, s)=$ $-\ln p(z, s)$. We can define our objective function as

$$
\begin{aligned}
\mathcal{L}_{\theta, \phi}(x, y, s) & =\lambda \mathbb{E}_{z \sim q_{\phi}}\left[\log p_{\theta}(x \mid z)\right] \\
& +\kappa \mathbb{E}_{z \sim q_{\phi}}\left[\log p_{\theta}(y \mid z)\right] \\
& -\rho \Gamma(z, s) .
\end{aligned}
$$

The full optimization problem becomes

$$
\left(\theta^{*}, \phi^{*}\right)=\underset{\theta, \phi}{\operatorname{argmax}} \mathcal{L}_{\theta, \phi}(x, y, s) .
$$

\subsection{Implementation}

The model is implemented as a Neural Network. Process of updating weights is implemented as a Stochastic Gradient Descent (SGD) [5]. To approximate 5, for a single point $x^{(d)}$, we take $V$ samples of $z$ and calculate

$$
\begin{aligned}
\hat{\mathcal{L}}_{\theta, \phi}\left(x^{(d)}, y^{(d)}, s^{(d)}\right) & =\frac{1}{V} \sum_{v=1}^{V}\left[\lambda \log p_{\theta}\left(x^{(d)} \mid z^{(d, v)}\right)\right. \\
& \left.+\kappa \log p_{\theta}\left(y^{(d)} \mid z^{(d, v)}\right)\right] \\
& -\rho \Gamma\left(z^{(d)}, s^{(d)}\right)
\end{aligned}
$$

Usually, we can just take a single sample $z^{(d)}$ for approximating expectation in 5. The full description is presented in the algorithm 2

\subsection{Latent space visualization}

Figure 13, shows latent space for DSLGMM model. Each point $z^{(d)}$ on the scatter plot is a sample from two dimensional $z \sim q_{\phi}(z \mid x)$. As we can see, classes are much better separated from each other than for both SVAE (see 


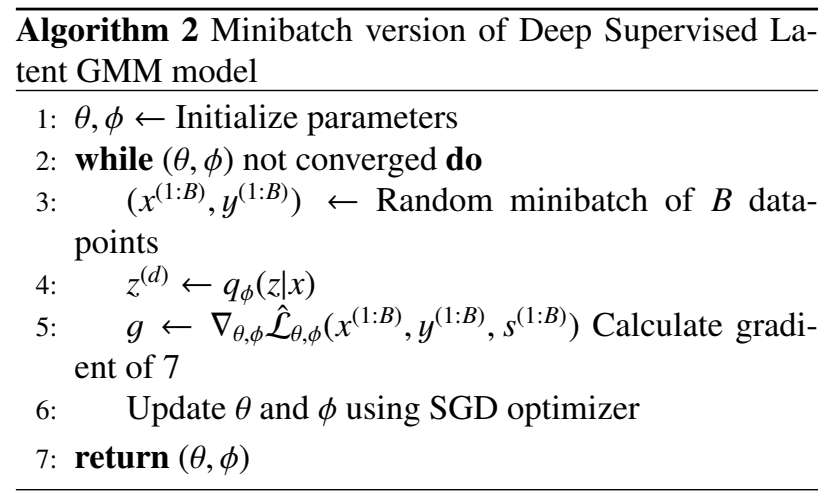

Fig. 4) and DLGMM (see Fig. 7). Additionally, DLGMM model sometimes produces latent space in which values of $z$ for two different classes are concentrated around a single Gaussian. It may also happen for DLGMM, that one or more classes end up in the center of coordinate system, that is, between all mixture components. There is no such problem with DSLGMM.

\section{Applications of DSLGMM model}

In this section, we introduce two applications of DSLGMM model. Firstly, our model can be used to define a better score for classification than the natural score $p_{\theta}(y \mid x)$. Secondly, DSLGMM model can be used to generate samples from a predefined class.

\subsection{Classifier with score}

We wish the classifier, to return confidence score for each prediction that it makes. This would allow us to choose some threshold value above which, our classifier makes little errors. For example, we could find a threshold value, above which our classifier makes no more that $2 \%$ of errors. For DSLGMM model, we can notice that samples from $z$ that are located near the center, are cases that we are most unsure of. Therefore, we can calculate the confidence of our classification as a norm $\|z\|$. Notice, that the choice of Gaussians parameters in GMM is crucial. If they were not located on a circle around the origin of coordinate, then the norm $\|z\|$ would not have been a good score for classification. For example, score calculated this way for VAE model would not work. To see that, compare visualizations of latent spaces in figures 4 and 13 .

\subsection{Generating new samples}

One of the useful applications of a generative model $p_{\theta}(x \mid z) p(z)$, is the ability of generating new, previously unseen, samples of data. In standard variational autoencoder, we generate point from $\mathcal{N}(0, I)$ and then push it through the decoder network, obtaining distribution $p_{\theta}(x \mid z)$. Since we are assuming, that our datasets contain information about classes and we have a way to supervise latent space (see section 4), we can train our model in such a way, that we can generate data from a single class. Results for perclass generated samples are shown in section 6.4.
Table 1: Dimensionality of datasets used for experiments

\begin{tabular}{ll}
\hline Dataset & Dimensionality \\
\hline MNIST & 784 \\
Poliblog & 1290 \\
SmsSpam & 16 \\
Reuters 2 & 2000 \\
Reuters 3 & 2000 \\
Reuters 5 & 2000 \\
\hline
\end{tabular}

\section{Experimental results}

In this section, we present results of some of the experiments with our models. We will start with the description of datasets used for those experiments (see Sect. 6.1). The rest is divided into three subsections, in which we show results for: comparison of performance of classifiers (see Sect. 6.2), classification score (see Sect. 6.3) and new samples generation (see Sect. 6.4).

\subsection{Datasets used for experiments}

We have used four datasets: first consists of 942 (742 train, 200 test), documents containing SMS labeled messages that have been extracted from SMS Spam Collection Data Set available on UCI Machine Learning Repository site and first introduced in [6]. The second dataset, is a collection of 773 (573 train, 200 test) political blogs available as part of the LDA R package [7]. The third dataset is a collection of documents that appeared on Reuters newswire in 1987 [8]. Reuters documents were assembled and indexed with categories. Original dataset consists of 22 different classes. We are using three subsets of them with 2, 3 and 5 classes, and call them reuters 2, reuters 3 and reuters 5 , respectively. The last dataset is not a collection of documents, but a MNIST dataset of handwritten digits [9]. Although this is not a text dataset, we have decided to include it in this work, because it will let us visualize some of the characteristics of the algorithm. All datasets were split to train and test datasets. Train datasets were used for training models. Final accuracy was measured on test datasets. Table 1 summarizes dimensionality of datasets used for the experiments.

\subsection{Experimental results for classification performance}

In figure 8, we present comparison of classification performance for various models. Each figure contains results for a single dataset. For each dataset, we have generated three additional datasets by training VAE, SVAE and DSLGMM and taking their latent spaces. For SVAE algorithm, hyperparameters in 1 were set to $\lambda=0, \kappa=1$ and $\rho=0.1$. The choice of those parameters is dictated by the following reasoning: $\lambda=0, \kappa=1$, because we are not interested in generating images, but only in classification. Parameter $\rho=0.1$, because we want to apply regularization, but we do not want it to be too strong. If $\rho$ were equal to 1 , then cost from $K L(q \| p)$ would outweigh cost that is responsible 
Table 2: Threshold values for $2 \%$ of errors

\begin{tabular}{ll}
\hline Dataset & Threshold \\
\hline MNIST & 0.42 \\
Poliblog & 1.04 \\
Reuters 2 & 0.62 \\
Reuters 3 & 0.81 \\
Reuters 5 & 1.12 \\
\hline
\end{tabular}

for classification $\log p_{\theta}(y \mid z)$. If, on the other hand, $\rho<0.1$, then regularization practically does not have any influence, and values of $z$ become large which leads to overfitting. For DSLGMM model, we have chosen the following parameters: $\lambda=0, \kappa=1$ and $\rho=1$. To original dataset and to three produced datasets, we have applied four classification algorithms: Random Forest, Decision Tree, Neural Network, Naive Bayes. Since SVAE is also a classifier, we include its accuracy as well. Results from SVAE will be marked as Embedded.

As can be observed in figure 8, SVAE works better for poliblog dataset. In all other cases, results obtained by running classification on data produced by DSLGMM outperform those produced by both VAE and SVAE. Another important fact is, that classifier run on this 2-dimensional representation produced by DSLGMM algorithm, achieves results comparable to those obtained on original dataset. Recall, that with the exception of smsSpam dataset, dimensionality of our datasets is usually counted in hundreds or thousands (see table 1).

\subsection{Experimental results for Latent Score}

In figure 9, we present results of applying score calculated as $\|z\|$, to five datasets described in section 6.1. We set values of hyperparameters in objective 5 , to $\lambda=0, \kappa=1$ and $\rho=1$. In the first picture (Left), we show how accuracy changes with respect to threshold. The accuracy is calculated only for documents, that meet $\|z\| \geq t$ for a given threshold value $t$. In the second picture (Right), we show how many points were classified for a given threshold $t$.

We can also calculate, how many cases can be classified for a given accuracy. This refers to the useful business application. For example, assume we would like to classify a blog as following republican or democratic view. It would be reasonable, to label only those for which our classifier makes little errors, e.g. less than 5\%. Looking at the Poliblog chart in figure 12, we can see, that we could classify about $25 \%$ or $10 \%$ entries for $\|z\|$ or $p_{\theta}(y \mid x)$ respectively. Clearly, in this case, score based on hidden variables $z$ is better. In table 2 and figure 10, we present threshold values, for which $98 \%$ of data in test datasets was correctly classified.

\subsection{Experimental results for generating new samples}

Figure 11 contains results of generating new data from one class at a time from MNIST model. There is ten rows, each
Table 3: Top eight words in data generated from first class of smsSpam model

\begin{tabular}{llll}
\hline $\begin{array}{c}\text { Top words } \\
\text { in first class }\end{array}$ & Sim. 1 & Sim. 2 & Sim. 3 \\
\hline can & can & will & will \\
now & will & can & just \\
will & just & just & can \\
just & now & now & now \\
call & call & call & call \\
get & get & get & get \\
send & send & send & send \\
new & free & free & stop \\
\hline
\end{tabular}

Table 4: Top eight words in data generated from second class of smsSpam model

\begin{tabular}{|c|c|c|c|}
\hline $\begin{array}{l}\text { Top words } \\
\text { in second class }\end{array}$ & Sim. 1 & Sim. 2 & Sim. 3 \\
\hline call & call & call & call \\
\hline free & free & free & free \\
\hline now & now & now & txt \\
\hline txt & txt & txt & now \\
\hline mobile & mobile & text & mobile \\
\hline text & claim & mobile & text \\
\hline stop & text & prize & stop \\
\hline claim & stop & stop & claim \\
\hline
\end{tabular}

for a single class. As we can see, we are able to capture the essence of the class and at the same time, the variability is still there. For example, if we look at the first row, we can say for sure that it is zero, but there are no two images exactly alike.

In the second experiment, we have generated three datasets from model trained for smsSpam dataset. In tables 3 and 4, we present top eight words for each class. We also include top eight words from original dataset. As we can see, we are able to capture per class word distribution very well.

Figure 5 and 6, contain results of similar experiments for poliblog dataset. This time however, the difference between classes is not that obvious. To make sure, that generated data really reflect true classes, we have trained random forest classifier on them, and tested the model on original test dataset. The accuracy was equal to 0.705 . The result is slightly worse than for original dataset, that was equal to 0.71 .

\section{Summary}

In this work, we have derived two new models called DLGMM and DSLGMM that are based on previous results in the area of Bayesian neural networks with hidden variables. We have proposed a different optimization objective than typically used, that allows us to shape latent space as a mixture of Gaussians. The first model finds latent space that is able to reflect different distributions associated with 
Table 5: Top five words in data generated from first class of poliblog model

\begin{tabular}{llll}
\hline $\begin{array}{l}\text { Top words } \\
\text { in first class }\end{array}$ & Sim. 1 & Sim. 2 & Sim. 3 \\
\hline just & just & just & just \\
obama & obama & obama & obama \\
clinton & like & like & like \\
said & people & people & people \\
like & dont & years & said \\
new & think & said & years \\
people & years & clinton & know \\
campaign & said & say & clinton \\
time & right & state & democratic \\
democratic & state & think & say \\
thats & democratic & republican & right \\
get & say & know & state \\
state & now & democratic & think \\
think & election & new & time \\
last & clinton & last & dont \\
years & time & dont & going \\
two & make & election & election \\
mccain & presidential & john & good \\
john & new & get & make \\
say & get & make & last \\
\hline
\end{tabular}

Table 6: Top five words in data generated from second class of poliblog model

\begin{tabular}{|c|c|c|c|}
\hline $\begin{array}{l}\text { Top words } \\
\text { in second class }\end{array}$ & Sim. 1 & Sim.n 2 & Sim. 3 \\
\hline obama & just & just & just \\
\hline just & like & like & obama \\
\hline people & obama & obama & like \\
\hline like & people & people & people \\
\hline said & democratic & democratic & think \\
\hline get & think & say & democratic \\
\hline barack & first & years & know \\
\hline read & said & think & say \\
\hline dont & going & said & years \\
\hline make & dont & know & dont \\
\hline know & years & time & time \\
\hline new & right & going & said \\
\hline time & election & right & first \\
\hline two & say & make & right \\
\hline democratic & now & candidate & candidate \\
\hline first & make & dont & make \\
\hline campaign & new & new & campaign \\
\hline last & time & house & new \\
\hline see & two & campaign & going \\
\hline mccain & president & first & barack \\
\hline
\end{tabular}
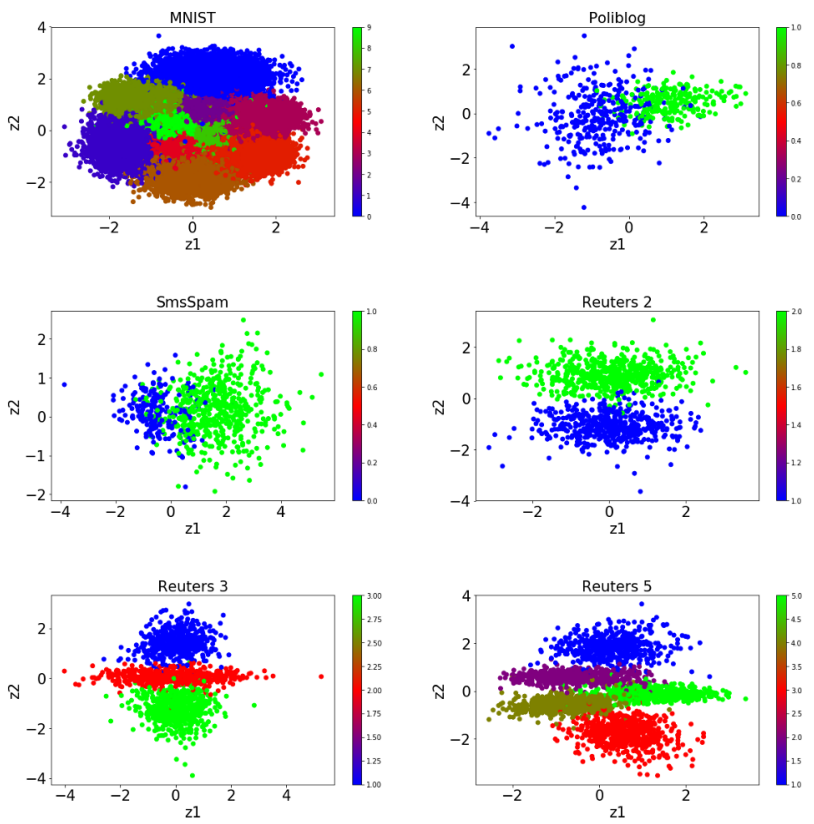

Figure 4: Latent space of Supervised VAE model for datasets described in Sec. 6.1
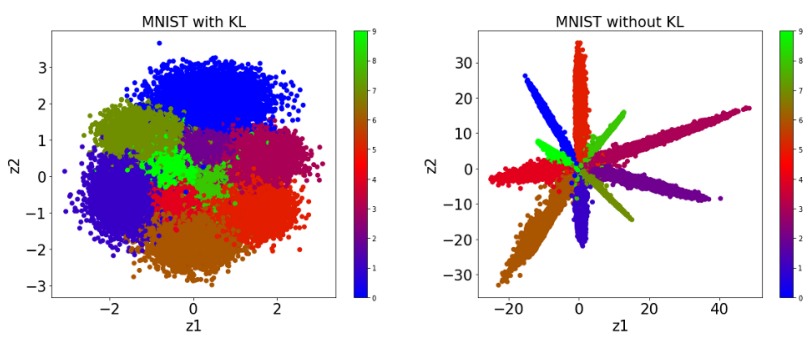

Figure 5: Latent space of Supervised VAE with and without regularizer. Figures were generated for 2-dimensional latent space.
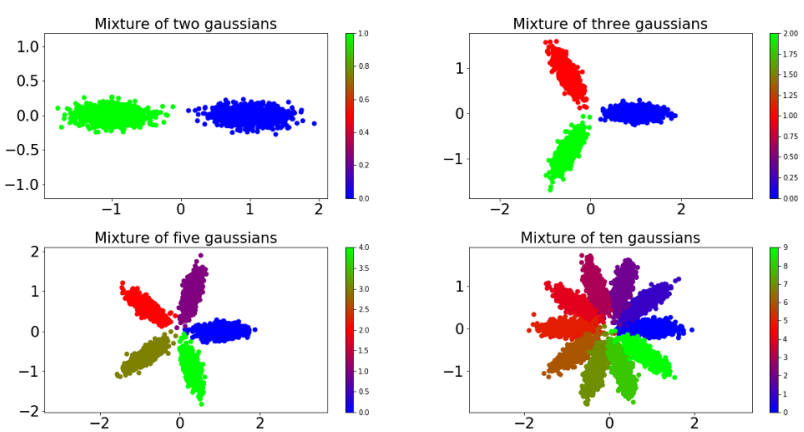

Figure 6: Synthetic data generated from mixtures of Gaussians, using parameters generated by algorithm 1

different classes. In the second model, we supervise this process by providing some permutation of labels to assign classes to mixture components. We have also presented two new applications: better score for classifier and ability to generate data for a given class. The second application is different from the original VAE model, where we have 

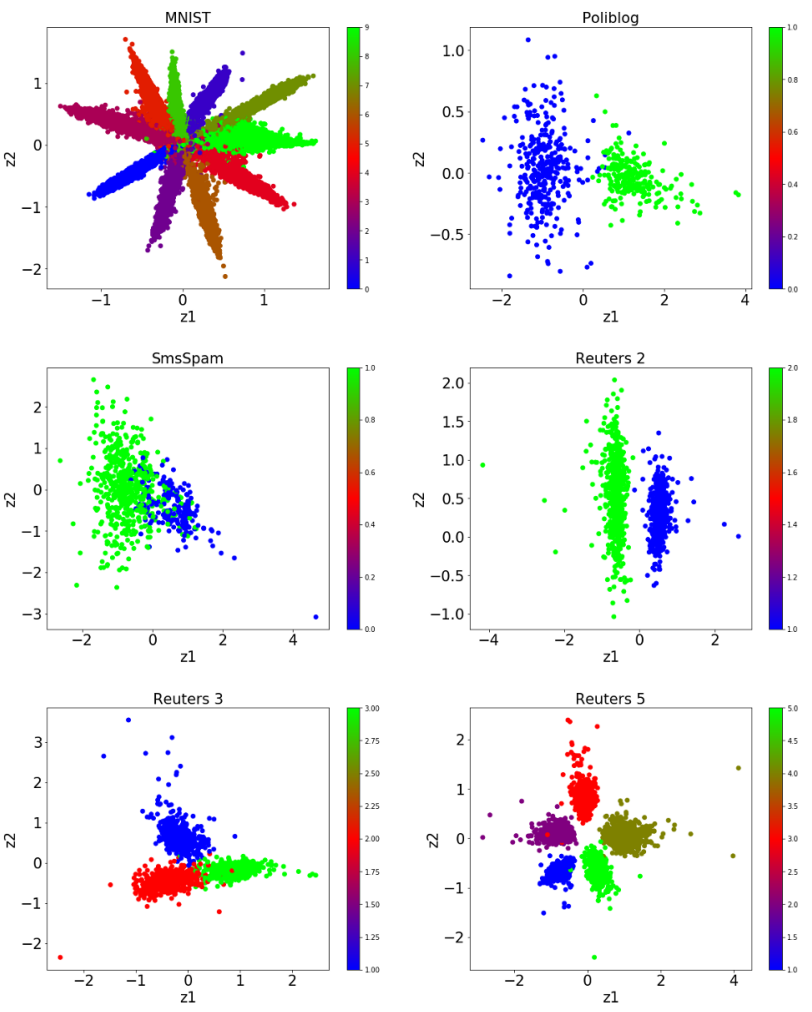

Figure 7: Latent space of Deep Generative Model with GMM latent space for train dataset
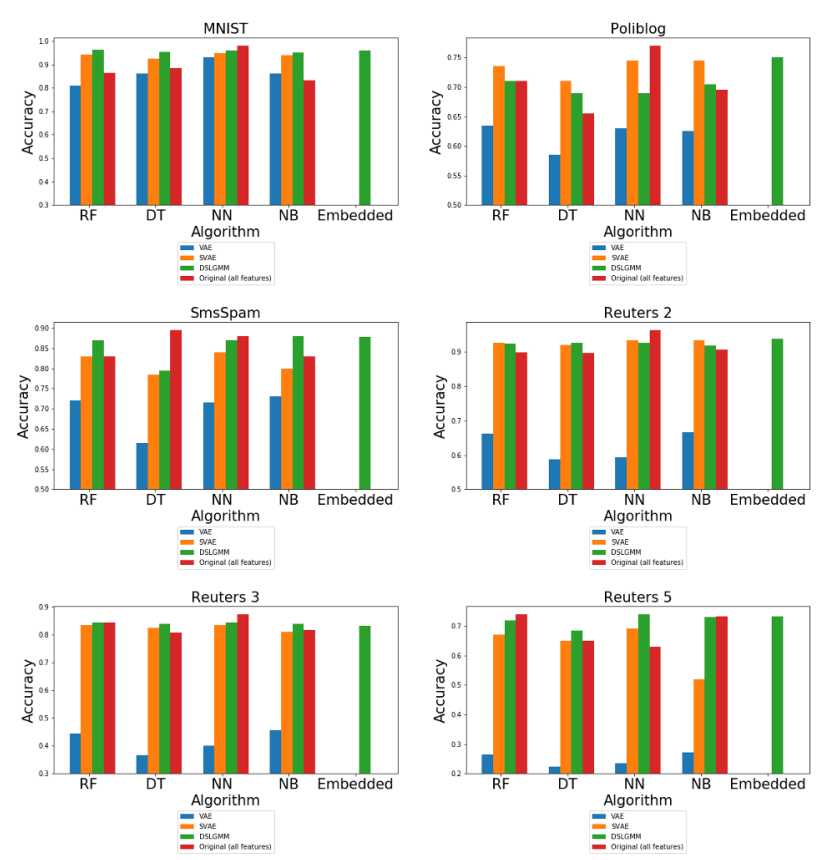

Figure 8: Comparison of VAE, SVAE and DSLGMM results for six datasets

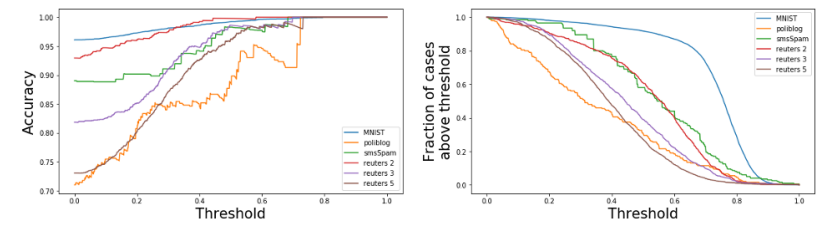

Figure 9: Classification for DSLGMM. (Left) presents Threshold vs. Accuracy. (Right) shows Threshold vs. Fraction of cases that are above the Threshold
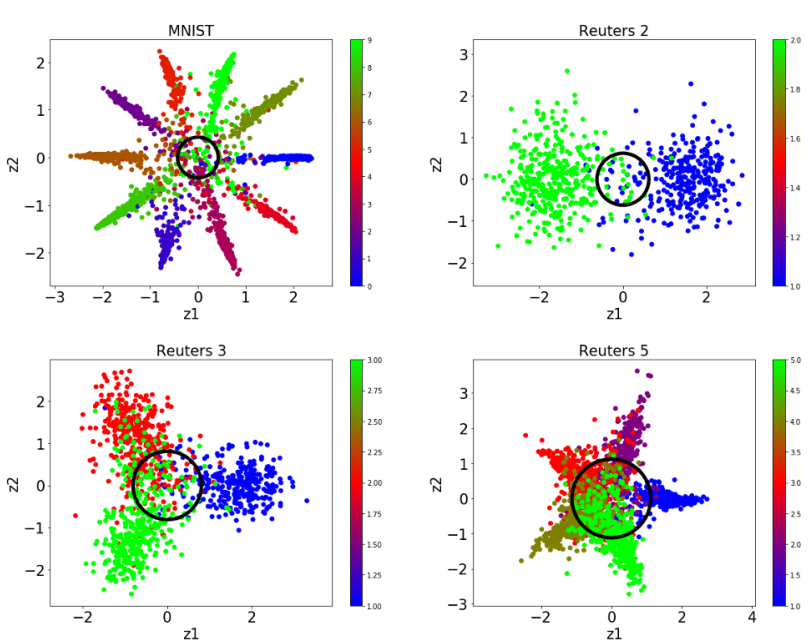

Figure 10: Latent space of test dataset with threshold for $98 \%$ accuracy for test dataset

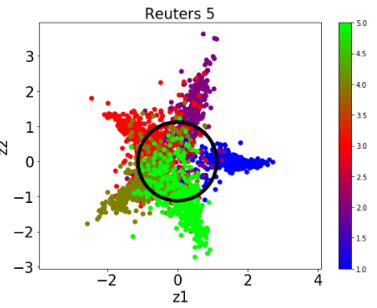

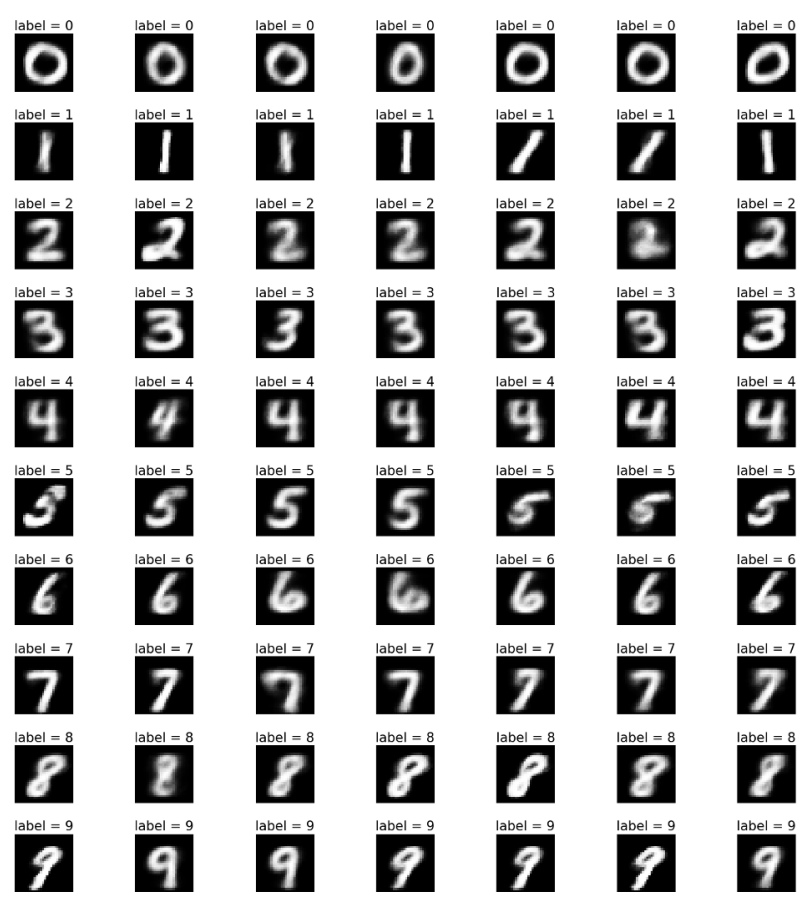

Figure 11: Generated digits 

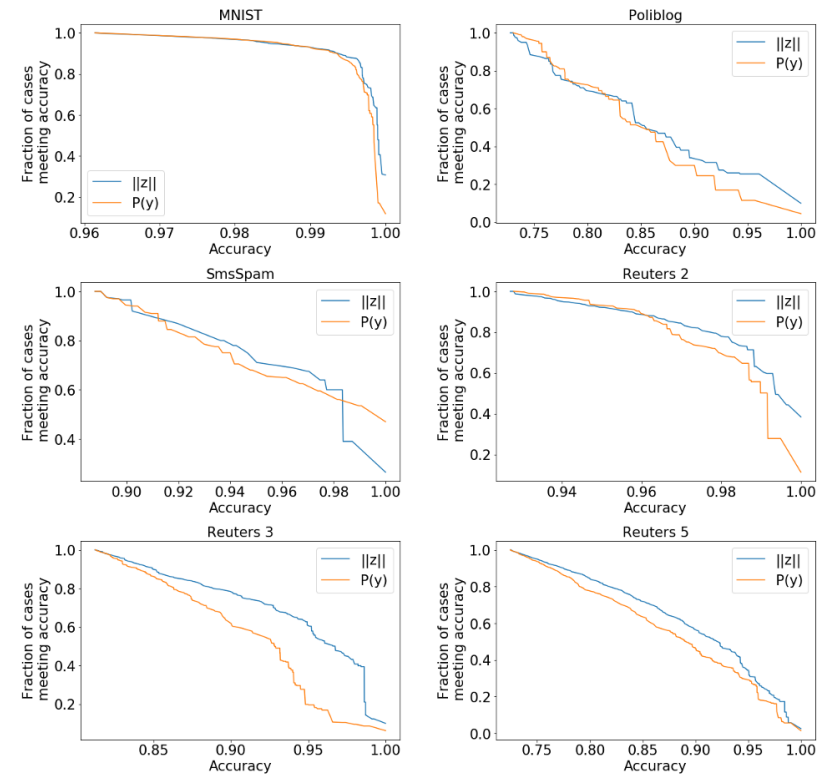

Figure 12: Comparison of scores calculated as $\|z\|$ and $p_{\theta}(y \mid x)$
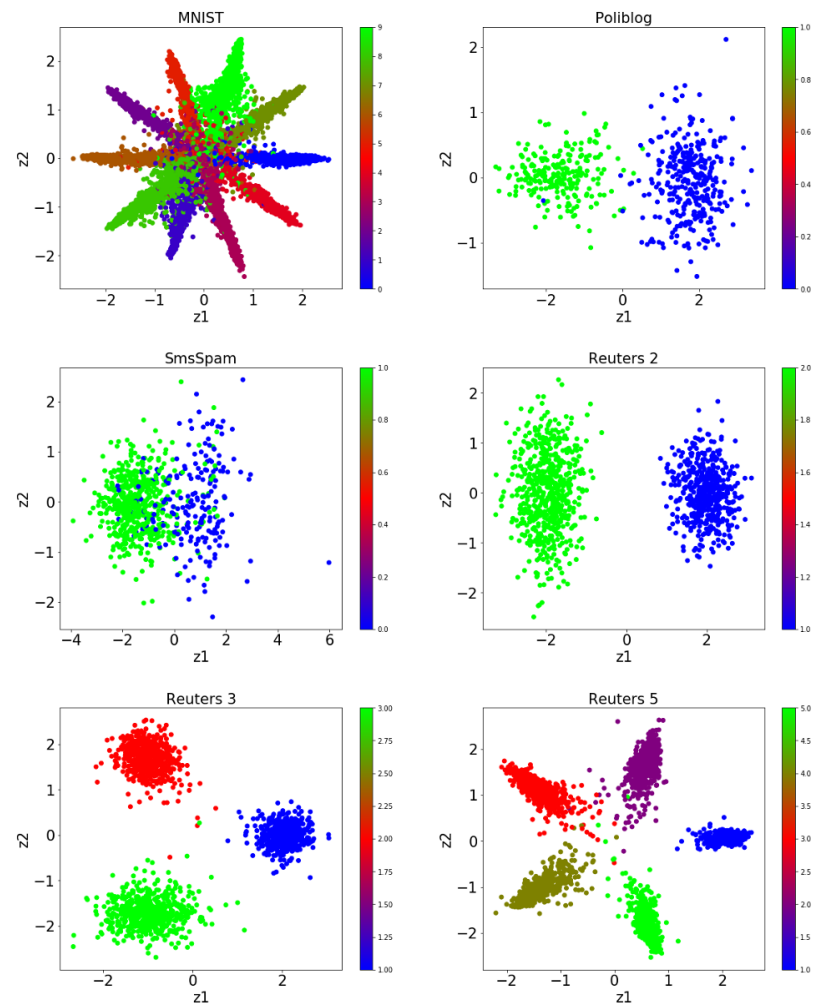

Figure 13: Latent space of Deep Generative Model with Supervised GMM latent space for train dataset no way of demanding from which class data is to be generated. We have shown in section 6 , that our approach gives good results.

\section{References}

[1] D.P. Kingma, M. Welling, arXiv preprint arXiv:1312.6114 (2013)

[2] D.J. Rezende, S. Mohamed, D. Wierstra, arXiv preprint arXiv:1401.4082 (2014)

[3] D.P. Kingma, D.J. Rezende, S. Mohamed, M. Welling, CoRR abs/1406.5298 (2014), 1406. 5298

[4] J. Gordon, J.M. Hernández-Lobato, arXiv preprint arXiv:1706.09751 (2017)

[5] H. Robbins, S. Monro, The annals of mathematical statistics pp. 400-407 (1951)

[6] T.A. Almeida, J.M.G. Hidalgo, A. Yamakami, Contributions to the study of SMS spam filtering: new collection and results, in Proceedings of the 11th ACM symposium on Document engineering (ACM, 2011), pp. 259-262

[7] J. Chang, lda: Collapsed Gibbs Sampling Methods for Topic Models (2015), r package version 1.4.2, https: //CRAN.R-project . org/package $=1$ da

[8] empty, Reuters-21578 dataset (empty), http://kdd.ics.uci.edu/databases/ reuters21578/reuters21578.html

[9] Y. LeCun, C. Cortes (2010) 